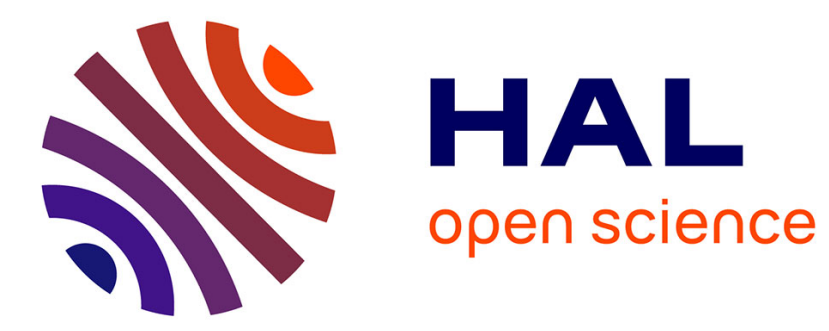

\title{
Benchmarking benchmarks: introducing new automatic indicators for benchmarking Spoken Language Understanding corpora
}

Frédéric Béchet, Christian Raymond

\section{- To cite this version:}

Frédéric Béchet, Christian Raymond. Benchmarking benchmarks: introducing new automatic indicators for benchmarking Spoken Language Understanding corpora. InterSpeech, Sep 2019, Graz, Austria. hal-02270633

\section{HAL Id: hal-02270633 \\ https://hal.science/hal-02270633}

Submitted on 26 Aug 2019

HAL is a multi-disciplinary open access archive for the deposit and dissemination of scientific research documents, whether they are published or not. The documents may come from teaching and research institutions in France or abroad, or from public or private research centers.
L'archive ouverte pluridisciplinaire HAL, est destinée au dépôt et à la diffusion de documents scientifiques de niveau recherche, publiés ou non, émanant des établissements d'enseignement et de recherche français ou étrangers, des laboratoires publics ou privés. 


\title{
Benchmarking benchmarks: introducing new automatic indicators for benchmarking Spoken Language Understanding corpora
}

\author{
Frédéric Béchet ${ }^{1}$, Christian Raymond ${ }^{2}$ \\ ${ }^{1}$ Aix Marseille Univ, Université de Toulon, CNRS, LIS, Marseille, France \\ ${ }^{2}$ INSA Rennes, IRISA, Rennes,France \\ frederic.bechetelis-lab.fr, christian.raymondeirisa.fr
}

\begin{abstract}
Empirical evaluation is nowadays the main evaluation paradigm in Natural Language Processing for assessing the relevance of a new machine-learning based model. If large corpora are available for tasks such as Automatic Speech Recognition, this is not the case for other tasks such as Spoken Language Understanding (SLU), consisting in translating spoken transcriptions into a formal representation often based on semantic frames. Corpora such as ATIS or SNIPS are widely used to compare systems, however differences in performance among systems are often very small, not statistically significant, and can be produced by biases in the data collection or the annotation scheme, as we presented on the ATIS corpus ("Is ATIS too shallow?, IS2018"). We propose in this study a new methodology for assessing the relevance of an SLU corpus. We claim that only taking into account systems performance does not provide enough insight about what is covered by current state-of-the-art models and what is left to be done. We apply our methodology on a set of 4 SLU systems and 5 benchmark corpora (ATIS, SNIPS, M2M, MEDIA) and automatically produce several indicators assessing the relevance (or not) of each corpus for benchmarking SLU models.

Index Terms: Spoken Language Understanding (SLU), benchmark, ATIS , SNIPS , M2M , MEDIA
\end{abstract}

\section{Introduction}

Spoken Language Understanding (SLU) consists in translating spoken transcriptions into a formal representation, often based on semantic frames. SLU received lately a particular attention as one of the crucial component of spoken chatbots and many models have been proposed to tackle this task although very few benchmark corpora are available to train and evaluate them. Corpora such as ATIS are widely used to compare systems, however differences in performance among systems are often very small, not statistically significant, and can be due to biases in the data collection or the annotation scheme, as we presented for the ATIS corpus [1].

We propose in this study a new methodology for assessing the relevance of an SLU corpus for benchmarking automatic tagging systems. We claim that only taking into account systems performance does not provide enough insight about what is covered by current state-of-the-art models and what is left to be done. We apply our methodology on a slot-tagging tasks for 4 benchmark corpora (ATIS, SNIPS, M2M, MEDIA). We build 4 tagging systems implementing 4 different machine learning models (Boosting, Conditional Random Fields (CRF), Multi-Layer Perceptron (MLP) and Recurrent Neural Networks (RNN) and automatically produce several indicators assessing the relevance (or not) of each corpus of the 4 corpus for benchmarking SLU models.

\section{Methodology}

The way SLU systems are benchmarked today consists mostly in a quantitative evaluation where the performance of each system is given on a corpus annotated with a semantic model (slot/value, frame, ...). These raw evaluations don't take into account the intrinsic characteristics of these test corpora. In other terms, can we assume that two corpora for which state-ofthe-art models achieve the same level of accuracy are comparable in terms of complexity and relevance for benchmarking SLU systems?

We believe that this is not the case as some corpora can have biases in the data collection process or the annotations provided, the intrinsic ambiguity of the semantic model chosen can be very different in each corpus (size of the semantic lexicon, average number of possible semantic label for each word) and finally the size of the training partition and its similarity toward the test partition in terms of lexical and semantic label distributions can also be very different. All these factors impact performance of a given SLU system, regardless of the model implemented to learn the task.

Describing corpora thanks to descriptive indicators about the semantic complexity of the annotation schemes or words and labels distributions is useful but this won't indicate which aspects are already well covered by current state-of-the-art models and which ones can still be considered as open issues. Moreover the discovery of corpus biases can only be done with respect to processing models, as a corpus characteristic becomes a bias only when it has an impact on the inference capabilities of the models. This is why we propose a new automatic methodology for assessing the relevance of a given corpus for benchmarking SLU systems based on the use of several SLU models, implementing different state-of-the-art paradigms. The first step in this methodology is to partition the test corpus into four clusters according to agreement/disagreement measures as well as correct/incorrect predictions obtained by all the SLU models trained on the task; then to train a classifier to automatically predict, for each sample in the test corpus, its cluster label. The relevance indicators for a given corpus are obtained from its cluster distribution as well as the capacity of the classifier to automatically predict cluster labels.

The application of this methodology on an SLU entity tagging task is described in the next sub-sections.

\subsection{Automatic clustering of test utterances}

We consider here an entity tagging task where the annotations are projected at the word label following a Begin, Inside, Outside scheme (B,I,O). The first step in our methodology is to develop a set of SLU systems $M=m_{1}, m_{2}, \ldots m_{n}$, implementing several inference models, trained on the same set of corpora $C_{1}, C_{2}, \ldots$, each corpora being split into a train $(C$-train $)$ 
and test $(C$-test $)$ partitions. For each word $w_{i}$ of an utterance $u \in C$-test, let label $(m, u, i)$ be the label predicted by model $m$ on $w_{i}$ and let label $(r e f, u, i)$ be the reference label of $w_{i}$. An example of this annotation scheme on the utterance $u=$ find flights arriving new-york new-york next saturday is given in table 1. Let's point out that choosing words as the basic units for semantic annotation has the advantage to be independent from a specific semantic model but has drawbacks since entity size impact the amount of errors.

\begin{tabular}{|c|c|c|c|c|c|}
\hline$i$ & word $w_{i}$ & label $($ ref, $u, i)$ & label $\left(m_{1}, u, i\right)$ & label $\left(m_{2}, u, i\right)$ & cluster \\
\hline 1 & find & O & O & O & AC \\
\hline 2 & flights & O & O & O & AC \\
\hline 3 & arriving & O & O & O & AC \\
\hline 4 & new-york & B-to-city & B-to-city & B-from-city & NC \\
\hline 5 & new-york & O & B-to-city & B-to-city & AE \\
\hline 6 & next & B-date-arr & B-date-dep & O & NE \\
\hline 7 & saturday & I-date-arr & I-date-dep & B-date-arr & NE \\
\hline
\end{tabular}

Table 1: Example of annotation of utterance $u$ with two SLU models $\left(m_{1}, m_{2}\right)$ and the resulting cluster for each word

We then partition all words in $C$-test according to two dimensions: agreement and correctness. If all systems agree, they belong to partition $\mathbf{A}$ (agreement), otherwise partition $\mathbf{N}$ (noagreement). If at least one system predict the correct label, they belong to partition $\mathbf{C}$ (correct), otherwise partition $\mathbf{E}$ for errors. This partitioning process produces 4 clusters formally defined as follows:

1. AC - Agreement/Correct: all labels label(ref,u,i) such as $\forall m \in M$, label $(m, u, i)=\operatorname{label}(\operatorname{ref}, u, i)$

2. AE - Agreement/Error: all labels label(ref,u,i) such as $\forall m, m^{\prime} \in M, \operatorname{label}(m, u, i)=\operatorname{label}\left(m^{\prime}, u, i\right)$ and $\operatorname{label}(m, u, i) \neq \operatorname{label}($ ref $, u, i)$

3. NC - No agreement/Correct: all labels label(ref,u,i) such as $\exists m, m^{\prime} \in M, \operatorname{label}(m, u, i) \neq \operatorname{label}\left(m^{\prime}, u, i\right)$ and $\operatorname{label}(m, u, i)=\operatorname{label}($ ref $, u, i)$

4. NE - No agreement/Error: all labels label(ref,u,i) such as $\exists m, m^{\prime} \in M, \operatorname{label}(m, u, i) \neq \operatorname{label}\left(m^{\prime}, u, i\right)$ and $\nexists k \in M, \operatorname{label}(k, u, i)=\operatorname{label}($ ref $, u, i)$

We then make the following assumptions on these clusters:

AC is the solved problem cluster, containing examples well covered by all models, regardless of their intrinsic performance.

$\mathrm{NC}$ is the system comparison cluster containing challenging examples covered by some state-of-the-art models (for example in line 4 of table 1 ).

$\mathbf{N E}$ is the open problem cluster containing examples not handled by any of the current models. This can correspond to two different situations: errors coming from a lack in the training data (such as Out-Of-Vocabulary words) or errors coming from real ambiguities not yet covered by current models.

$\mathbf{A E}$ is the annotation problem cluster containing mostly annotation errors or biases in the training corpus. We consider that if all systems make the same errors, it might come from a mistake in the annotation process, like line 6 of table 1, where the repetition of the word new-york is erroneously labelled as $O$ in the reference annotation. Or it might come from a missed entity by all models, due like cluster $\mathbf{N E}$ to a lack in the training data.

We believe that the word distribution among the 4 clusters for a given corpus $C$ provides good insights about the relevance of $C$ for benchmarking systems. We propose to define our first indicator of corpus complexity as $I_{1}=100-\frac{|A C| \times 100}{\mid C \text {-test } \mid}$. A low value of $I_{1}$ indicates that the corpus is nearly a solved problem as most examples are correctly labelled by all models, even the low performing ones, therefore this corpus not necessarily relevant to compare different SLU models performance.

\subsection{Automatic prediction of cluster labels}

Once the clustering into the four clusters has been done, we want to check how efficient would be a classifier to automatically classify each word of the test corpus as belonging to AC, $\mathrm{AE}, \mathrm{NC}$ and NE thanks only to corpus characteristics. Our assumption is that cluster prediction accuracy for error clusters $\mathrm{AE}$ and NE are good indicators of the kind of errors contained in them:

$\mathrm{NE}$ : we consider that the examples in NE which are not properly classified correspond to the real challenging examples (not predictable). In contrast to the predictable examples that can correspond to error regularities that can be easily fixed, for example by incorporating knowledge about the task (list of cities, movies, restaurant,etc.) coming from the task database.

$\mathrm{AE}$ : the examples that can be accurately classified as belonging to $\mathrm{AE}$ must correspond to a corpus bias rather than an intrinsic ambiguity.

To train this classifier (called $C C$ ) we build a training corpus where each word $w_{i}$ of an utterance $u \in C$-test is a training example, with features related to its left and right context as well as its label. The class to predict $C C(u, i)=c$ is the cluster $i d$ the word $w_{i}$ in $u$ belongs to. For example, for the word 5 of table 1 , we generate the following training example:

left (arriving, o new-york, to-city)

word (new-york), label (0)

right (next, date-arr saturday, date-arr) $\Rightarrow \mathrm{AE}$

We train the classifier following a 10-fold setting and compute precision $(\mathrm{P})$, recall $(\mathrm{R}), \mathrm{F}$-measure $(\mathrm{F})$ as well as the global classification error rate. F-measure for clusters NE and $\mathrm{AE}$ can be used to find how important is the impact of biases in the classification process. We define our second corpus complexity indicator $I_{2}$ as being the classification error rate on the four clusters.

It is defined as: $I_{2}=100-\frac{\mid \forall u \forall i C C(u, i)=\text { error } \mid}{\mid C \text {-test } \mid} \times 100$

A low value for $I_{2}$ indicates that the corpus is very predictable in terms of error prediction, therefore with little interest for evaluating systems. At the contrary a high value for $I_{2}$ is a good indicator of corpus complexity.

\section{Application}

We apply our methodology on a set of five SLU corpora on an entity tagging task, using four different tagging models for estimating agreement and correct/incorrect predictions. They are presented in the next subsections.

\subsection{SLU corpora}

We did experiments on 5 standard benchmarks used to evaluate slot tagging systems whose characteristics are presented in table 2:

1. M2M: this corpus is a fusion of two datasets containing dialogues for restaurant and movie ticket booking. It has been released by [2] and collected using their M2M 


\begin{tabular}{|l|r|r|r|r|r|}
\hline corpus & ATIS & MEDIA & SNIPS & SNIPS70 & M2M \\
\hline \hline vocabulary & 1117 & 2445 & 14354 & 4751 & 900 \\
\hline \#tags & 84 & 70 & 39 & 39 & 12 \\
\hline train size & 4978 & 12908 & 13784 & 2100 & 8148 \\
\hline test size & 893 & 3005 & 700 & 700 & 4800 \\
\hline av. turn length & $11[2,42]$ & $8[2,193]$ & $10[3,36]$ & & $7[2,30]$ \\
\hline
\end{tabular}

Table 2: Corpus characteristics

framework (Machines Talking To Machines) that combines dialogue self-play and crowd sourcing to generate dialogues.

2. ATIS: The Air Travel Information System (ATIS) task [3] is dedicated to provide flight information. The training set consists of 4978 utterances selected from the Class A (context independent) training data in the ATIS2 and ATIS-3 corpora while the ATIS test set contains both the ATIS-3 NOV93 and DEC94 datasets. The version is the widely used corrected version released with [4].

3. MEDIA: this corpus is made of 1250 French dialogue, dedicated to provide tourist information. It has been collected by ELDA, following a Wizard of $\mathrm{Oz}$ protocol: 250 speakers have followed 5 hotel reservation scenarios. This corpus has been transcribed manually and annotated with concepts from a rich semantic ontology [5].

4. SNIPS: this corpus has been collected by the SNIPS company. It is dedicated to 7 in-house tasks, SearchCreativeWork, GetWeather, BookRestaurant,PlayMusic, AddToPlaylist,RateBook, SearchScreeningEvent [6].

5. SNIPS70: The SNIPS benchmark is proposed in two configurations. SNIPS70 is the same as the previous one but the training set is limited to 70 queries per intent, randomly chosen, reflecting the fact that in a real life scenario, even an enthusiastic NLU developer will generally stop after supervising around 70 query examples. This makes the training set more close to real NLU development condition.

\subsection{SLU models}

We developed four different entity tagging systems, each of them implementing a different machine-learning model with different characteristics:

1. Boost: a boosting algorithm of small decision trees called bonsai trees [7]. This is a very efficient text classifier but is not dedicated to model sequence-to-sequence problems. It does not model either any output label dependencies. We used 1000 bonsai trees of size 2 (4 leaves) on word 1-grams with their relative position. Since the classifier is performing feature selection, the feature window cover the full utterance.

2. CRF: a standard CRF algorithm with symbolic input features, very relevant to model output label dependencies. It uses a feature set of 1-grams word/relative positions in an observation windows of size 7 around the current decision step.

3. MLP: a standard single-hidden-layer feed-forward neural network with an hidden layer of $100 \mathrm{ReLu}$ neurons and a joint embedding layer of size 100; it uses word embedding representations but does not model any target label dependencies.
4. BiGru+CRF: a bidirectional recurrent GRU [8] network used to encode the sequence of words into a vector, followed by a CRF output layer. It implements a 200 $(2 * 100)$ encoded utterance representation. This is the most elaborate model, with word embedding, it has access to the whole utterance thanks to the GRU and model target label dependencies thanks to the CRF objective. However this model is the one with the highest number of parameter.

In all our experiments we did not use any pretrained embeddings or any knowledge base such as named entity dictionnaries since the goal here is not to obtain the best tagging accuracy but to observe decisions agreement among systems trained exactly in the same conditions. All neural based models are build using Keras [9], bonzaiboost implementation has been used for boosting [7] and Wapiti [10] for symbolic CRF. The model selection strategy for neural systems is to keep the best set of parameters among 50 epochs according to the validation set (or training set if no official validation set is provided); regularisation is done using a dropout [11] of 0.5 at the output of the last -1 layer of the network.

Table 4 reports performance for each model on each corpus using the global F-measure F1 computed by the connlleval scoring script. Note that no system is particularly tuned for a given corpus therefore small differences between systems are not necessarily significant. Unsurprisingly F1 obtained on most corpora are lower than those reported in the literature since no pretrained embeddings or semantic lexicon were used.

We can note, that the best F1 obtained on ATIS, M2M and SNIPS are pretty similar, around $94 \%$. On the other hand performance on MEDIA and SNIPS70 are much lower, around $86 \%$. If all methods perform equally well on M2M and ATIS, this is not the case for MEDIA and SNIPS: for MEDIA, using a classifier with a bag of features, not modelling any sequence as in Boost, is a big handicap, leading to poor results $(-15 \%$ compared to the best one); for SNIPS and SNIPS70, not modelling output label dependencies like Boost or MLP has a great negative impact on performance (nearly $-10 \%$ ). For SNIPS70, not having enough data to train a model with a lot of parameter such as BiGru+CRF has also a negative impact.

\subsection{Estimating indicator $I_{1}$ and $I_{2}$}

Table 3 show the size of the 4 clusters AC, AE, NC, NE we obtained following our methodology along with their 5 most frequent misrecognized labels. By computing our indicator $I_{1}$ from the size of cluster $\mathrm{AC}$, as presented in section 2.1, we can compare each corpus in a more accurate way than just looking at the $F 1$ max obtained by the best system, as presented in table 6 . As we can see, although ATIS, M2M and SNIPS obtain roughly the same F1 max, they differ in terms of $I_{1}$, ATIS being the most solved corpora compared to the other two. Similarly $I_{1}$ emphasises the difference between SNIPS70 and MEDIA.

We then generate a corpus in order to train a model for classifying word/label tokens as $\mathrm{AC}, \mathrm{AE}, \mathrm{NC}, \mathrm{NE}$ thanks only to word and label features from the utterance transcription, as presented in section 2.2. As input features we provided, the reference label, the word itself, the left and right word context, the fact that the word is an Out-Of-Vocabulary words with respect to the training corpus, the number of potential labels for this word in the train and the utterance length. The classifier we use is the Bonzai Boost classifier presented in the previous section, trained with a 10-fold process on each test corpus of our 5 corpora. Table 5 shows the prediction accuracy of this classifier for 


\begin{tabular}{|c|c|c|c|c|c|c|c|c|c|c|}
\hline & \multicolumn{2}{|l|}{ M2M } & \multicolumn{2}{|l|}{ ATIS } & \multicolumn{2}{|l|}{ MEDIA } & \multicolumn{2}{|c|}{ SNIPS } & \multicolumn{2}{|c|}{ SNIPSs70 } \\
\hline & 5632 samples & $83.2 \%$ & 2472 samples & $88.3 \%$ & 11693 samples & $69.6 \%$ & 2589 samples & $81.2 \%$ & 1947 samples & $61.1 \%$ \\
\hline \multirow{5}{*}{$\mathrm{AC}$} & time & $26.0 \%$ & toloc-city_name & $27.7 \%$ & reponse & $12.7 \%$ & object_name & $16.4 \%$ & object_name & $13.5 \%$ \\
\hline & date & $13.4 \%$ & fromloc-city_name & $27.1 \%$ & command-tache & $11.8 \%$ & playlist & $9.4 \%$ & object_type & $9.6 \%$ \\
\hline & theatre_name & $12.1 \%$ & depart_date-day_name & $8.3 \%$ & temps-date & $9.2 \%$ & timeRange & $8.3 \%$ & playlist & $9.3 \%$ \\
\hline & restaurant_name & $12.0 \%$ & depart_time-periodofday & $4.5 \%$ & nombre & $7.1 \%$ & object_type & $7.5 \%$ & timeRange & $7.6 \%$ \\
\hline & location & $10.5 \%$ & airline_name & $4.0 \%$ & localisation-ville & $6.3 \%$ & artist & $5.0 \%$ & spatial_relation & $5.6 \%$ \\
\hline \multirow{6}{*}{$\mathrm{NC}$} & 310 samples & $4.6 \%$ & 229 samples & $8.2 \%$ & 3702 samples & $22.0 \%$ & 540 samples & $16.9 \%$ & 1112 samples & $34.9 \%$ \\
\hline & movie & $50.3 \%$ & fromloc-city_name & $9.6 \%$ & command-tache & $9.6 \%$ & object_name & $18.5 \%$ & object_name & $23.2 \%$ \\
\hline & restaurant_name & $34.8 \%$ & aircraft_code & $7.0 \%$ & objet & $8.3 \%$ & artist & $15.9 \%$ & artist & $13.8 \%$ \\
\hline & num_tickets & $3.9 \%$ & city_name & $4.8 \%$ & nom & $7.1 \%$ & movie_name & $11.3 \%$ & timeRange & $9.4 \%$ \\
\hline & rating & $3.2 \%$ & arrive_date-day_name & $4.4 \%$ & localisation-ville & $5.5 \%$ & playlist & $9.3 \%$ & playlist & $8.8 \%$ \\
\hline & time & $2.9 \%$ & airport_name & $4.4 \%$ & local.-lieuRelatif-gen. & $4.9 \%$ & timeRange & $6.5 \%$ & movie_name & $6.7 \%$ \\
\hline \multirow{6}{*}{$\mathrm{AE}$} & 687 samples & $10.2 \%$ & 63 samples & $2.3 \%$ & 804 samples & $4.8 \%$ & 30 samples & $0.9 \%$ & 28 samples & $0.9 \%$ \\
\hline & restaurant_name & $54.6 \%$ & city_name & $34.9 \%$ & command-tache & $20.4 \%$ & artist & $23.3 \%$ & movie_name & $17.9 \%$ \\
\hline & movie & $40.9 \%$ & depart_time-periodofday & $12.7 \%$ & objet & $16.4 \%$ & entity_name & $23.3 \%$ & artist & $14.3 \%$ \\
\hline & num_tickets & $3.8 \%$ & fromloc-city_name & $11.1 \%$ & connectProp & $6.6 \%$ & album & $16.7 \%$ & object_name & $14.3 \%$ \\
\hline & rating & $0.3 \%$ & toloc-city_name & $9.5 \%$ & command-dial & $6.5 \%$ & playlist & $10.0 \%$ & city & $10.7 \%$ \\
\hline & location & $0.1 \%$ & airport_name & $7.9 \%$ & reponse & $5.3 \%$ & object_select & $6.7 \%$ & entity_name & $10.7 \%$ \\
\hline \multirow{6}{*}{ NE } & 137 samples & $2.0 \%$ & 34 samples & $1.2 \%$ & 604 samples & $3.6 \%$ & 35 samples & $1.1 \%$ & 107 samples & $3.4 \%$ \\
\hline & movie & $56.9 \%$ & state_name & $23.5 \%$ & objet & $17.2 \%$ & album & $22.9 \%$ & album & $18.7 \%$ \\
\hline & restaurant_name & $40.9 \%$ & airport_code & $14.7 \%$ & local.-lieuRelatif-gen. & $6.5 \%$ & state & $11.4 \%$ & playlist & $15.9 \%$ \\
\hline & time & $1.5 \%$ & city_name & $11.8 \%$ & command-tache & $6.1 \%$ & timeRange & $11.4 \%$ & country & $9.3 \%$ \\
\hline & location & $0.7 \%$ & aircraft_code & $8.8 \%$ & chambre-equipement & $5.5 \%$ & track & $11.4 \%$ & & $8.4 \%$ \\
\hline & & & airport_name & $8.8 \%$ & nom & $5.3 \%$ & entity_name & $11.4 \%$ & state & $6.5 \%$ \\
\hline
\end{tabular}

Table 3: Label distribution according to the 4 clusters for each corpora

\begin{tabular}{|l|r|r|r|r|r|}
\hline F-measure & M2M & ATIS & MEDIA & SNIPS & SNIPS70 \\
\hline Boost & 92.6 & $\mathbf{9 4 . 2}$ & 70.6 & 87.2 & 78.1 \\
\hline MLP & $\mathbf{9 3 . 1}$ & 93.2 & 82.3 & 85.7 & 75.9 \\
\hline CRF & 91.7 & 92.4 & $\mathbf{8 5 . 7}$ & $\mathbf{9 4 . 0}$ & $\mathbf{8 7 . 9}$ \\
\hline BiGru+CRF & 92.5 & 93.9 & 85.6 & 91.8 & 74.1 \\
\hline
\end{tabular}

Table 4: Tagging performance for all systems on all corpora in terms of Micro F1 using conlleval scoring script (in \%)

each corpora and cluster.

As we can see classification accuracy varies a lots according to the cluster and the corpus considered. These results are especially interesting for the error clusters (AE and NE), because a high or low prediction score for these clusters allows us to further characterize them as mentioned before.

For NE, a high predictability score suggests that errors in this corpus are recurrent and identifiable. Indeed, NE from corpus $\mathrm{M} 2 \mathrm{M}$ is highly predictable, and as we can see in table 3 is composed of errors in movie and restaurant name labels. An examination of these utterances showed us that they had no context to help disambiguate the expression when the restaurant or movie name have not been seen in the training conditions. By adding the list of restaurant and movie names to the tagging models these errors will move from NE to AC cluster. It is partly true for ATIS where the predictability is medium and where the main errors are state_name, airport_name and code that can be easily incorporated into the models.

Once again we have a high predictability score for $\mathrm{AE}$ in $\mathrm{M} 2 \mathrm{M}$ and medium in ATIS while the main errors in this cluster in M2M are the same as NE, so same conclusion can be drawn. In ATIS the main error in this cluster is on "city name" and as shown in [1] there are two issues on this label, the first one is frequent annotation errors when a user mention twice the same city-name, only the first occurrence is labelled; the second one is errors on ground transportation queries that were not included in the semantic annotation scheme.

By looking at the indicator $I_{2}$ (cluster classification error rate), as shown in table 6 , we can have an additional view on corpora complexity: although M2M seems to be more challenging than ATIS according to $I_{1}$ it is almost completely predictable in terms of cluster classification according to $I_{2}$. This means that the errors in M2M are due to missing data from the training corpus, and not internal complexity. Similarly $I_{1}$ indicates than SNIPS70 is more difficult than MEDIA, but by looking at $I_{2}$ we draw the conclusion than MEDIA is intrinsically more ambiguous than SNIPS70. In all cases $I_{1}$ and $I_{2}$ are much more informative than F1max.

\begin{tabular}{|c|r|r|r|r|r|}
\hline & M2M & \multicolumn{1}{|c|}{ ATIS } & MEDIA & SNIPS & SNIPS70 \\
\hline AC & 99.8 & 98.6 & 89.1 & 95.4 & 92.5 \\
\hline NC & 71.0 & 60.8 & 45.5 & 49.9 & 64.6 \\
\hline AE & 89.7 & 55.1 & 27.5 & 22.2 & 0.0 \\
\hline NE & 71.2 & 45.9 & 18.0 & 12.8 & 32.7 \\
\hline \hline All & $\mathbf{9 9 . 1}$ & $\mathbf{9 7 . 0}$ & $\mathbf{7 9 . 5}$ & $\mathbf{9 1 . 1}$ & $\mathbf{8 6 . 5}$ \\
\hline \hline error rate & 0.9 & 3.0 & 20.5 & 8.9 & 13.6 \\
\hline
\end{tabular}

Table 5: Cluster predictability in F1 and classification error rate for all corpus

\begin{tabular}{|c|r|r|r|r|r|}
\hline Indicators & M2M & \multicolumn{1}{|c|}{ ATIS } & MEDIA & SNIPS & SNIPS70 \\
\hline max F1 & 93.1 & 94.2 & 85.7 & 94.0 & 87.9 \\
\hline$I_{1}$ & 16.8 & 11.7 & 30.4 & 18.8 & 38.9 \\
\hline$I_{2}$ & 0.9 & 3.0 & 20.5 & 8.9 & 13.6 \\
\hline
\end{tabular}

Table 6: Indicators max Fl, $I_{1}$ and $I_{2}$ on the 5 corpora

We claim that the predictability of a cluster measure the overall simplicity of a corpus: the more a cluster is predictable, the more examples are similar or contains similar patterns than can be learned easily. Therefore indicator $I_{2}$ is a good indicator of the quality of a corpus to be used as a benchmark. According to our experiments and the indicators in table 6 , we may rank these 5 benchmarks from the most challenging one to the almost-solved one for evaluating slot tagging systems as: $\mathrm{MEDIA} \rightarrow$ SNIPS70 $\rightarrow$ SNIPS $\rightarrow$ ATIS $\rightarrow$ M2M.

\section{Conclusion}

We proposed in this study a new methodology for assessing the relevance of an SLU corpus based on two indicators, $I_{1}$ and $I_{2}$, obtained through the study of firstly the agreement among several SLU systems developed for the task and secondly the predictability of the error classes made by the different systems. We apply our methodology on a set of 4 SLU systems and 5 benchmark corpora obtaining a ranking of these corpora from the most ambiguous to the almost-solved one that we claim is more accurate than just using the F1 max score. 


\section{References}

[1] F. Béchet and C. Raymond, "Is ATIS too shallow to go deeper for benchmarking Spoken Language Understanding models?" in InterSpeech 2018, Hyderabad, India, Sep. 2018, pp. 1-5. [Online]. Available: https://hal.inria.fr/hal-01835425

[2] P. Shah, D. Hakkani-Tür, G. Tür, A. Rastogi, A. Bapna, N. Nayak, and L. Heck, "Building a conversational agent overnight with dialogue self-play," arXiv preprint arXiv:1801.04871, 2018.

[3] D. A. Dahl, M. Bates, M. Brown, W. Fisher, K. Hunicke-Smith, D. Pallett, C. Pao, A. Rudnicky, and E. Shriberg, "Expanding the scope of the ATIS task: the ATIS-3 corpus," in HLT, 1994, pp. $43-48$.

[4] C. Raymond and G. Riccardi, "Generative and Discriminative Algorithms for Spoken Language Understanding," in InterSpeech, Antwerp, Belgium, August 2007, pp. 1605-1608.

[5] H. Bonneau-Maynard, S. Rosset, C. Ayache, A. Kuhn, and D. Mostefa, "Semantic Annotation of the French Media Dialog Corpus," in InterSpeech, Lisbon, September 2005. [Online] Available: ftp://tlp.limsi.fr/public/IS052010.PDF

[6] A. Coucke, A. Saade, A. Ball, T. Bluche, A. Caulier, D. Leroy, C. Doumouro, T. Gisselbrecht, F. Caltagirone, T. Lavril, M. Primet, and J. Dureau, "Snips voice platform: an embedded spoken language understanding system for private-by-design voice interfaces," CoRR, vol. abs/1805.10190, 2018. [Online]. Available: http://arxiv.org/abs/1805.10190

[7] A. Laurent, N. Camelin, and C. Raymond, "Boosting bonsai trees for efficient features combination : application to speaker role identification," in InterSpeech, Singapour, September 2014. [Online]. Available: http://bonzaiboost.gforge.inria.fr

[8] K. Cho, B. van Merrienboer, Ç. Gülçehre, F. Bougares, H. Schwenk, and Y. Bengio, "Learning phrase representations using RNN encoder-decoder for statistical machine translation," CoRR, vol. abs/1406.1078, 2014. [Online]. Available: http://arxiv.org/abs/1406.1078

[9] F. Chollet et al., "Keras," https://github.com/keras-team/keras, 2015.

[10] T. Lavergne, O. Cappé, and F. Yvon, "Practical Very Large Scale CRFs," in Proceedings the 48th Annual Meeting of the Association for Computational Linguistics (ACL). Association for Computational Linguistics, July 2010, pp. 504-513. [Online]. Available: http://www.aclweb.org/anthology/P10-1052

[11] G. E. Hinton, N. Srivastava, A. Krizhevsky, I. Sutskever, and R. Salakhutdinov, "Improving neural networks by preventing co-adaptation of feature detectors," CoRR, vol. abs/1207.0580, 2012. [Online]. Available: http://arxiv.org/abs/1207.0580 